\title{
FINITE TRAVELLING WAVES FOR SEMILINEAR PARABOLIC SYSTEMS
}

\author{
by MING-XIN WANG*
}

(Received 28th January 1997)

In this paper, finite travelling waves for the semilinear parabolic systems

$$
u_{i t}=d_{i} u_{i x x}-e_{i} \prod_{j=1}^{n} u_{j}^{m_{i j}}, i=1, \ldots, n
$$

are studied, where $d_{i}>0, e_{i}>0, m_{i j} \geq 0$ for all $1 \leq i, j \leq n$, and $\sum_{j=1}^{n} m_{i j}>0$ for all $1 \leq i \leq n$. Let $M=\left(m_{i j}\right)_{n \times n}$ and $A=I-M$. It will be proved that $(*)$ has finite travelling waves if and only if all principal minors of $A$ are positive. Moreover, some asymptotic behaviours of finite travelling waves will be obtained.

1991 Mathematics subject classification: $35 \mathrm{~K} 55,35 \mathrm{~K} 57,35 \mathrm{~K} 60$.

\section{Introduction and main results}

In this paper, finite travelling waves (FTW) of the semilinear parabolic systems

$$
u_{i t}=d_{i} u_{i x x}-e_{i} \prod_{j=1}^{n} u_{j}^{m_{i j}}, x \in \mathbb{R}^{1}, t>0, i=1, \ldots, n
$$

are studied, where $d_{i}>0, e_{i}>0, m_{i j} \geq 0, i, j=1, \ldots, n$, and $\sum_{j=1}^{n} m_{i j}>0, i=1, \ldots, n$. By a travelling wave of (1) with speed $c$, we mean a solution of (1) of the form

$$
u_{i}(x, t)=y_{i}(z), z=x+c t, c \in \mathbb{R}^{1}, i=1, \ldots, n,
$$

where $y_{i}(z)$ are nonnegative and nontrivial, and $y_{i}(z) \rightarrow 0$ as $z \rightarrow-\infty$. If there exists a finite $z_{0} \in \mathbb{R}^{\prime}$ such that $y_{i}(z) \equiv 0$ for $z \leq z_{0}, i=1, \ldots, n$, we say that $\left(y_{1}(z), \ldots, y_{n}(z)\right)$ is a finite travelling wave (FTW). Owing to the invariance property of travelling waves under translation, it is easy to see that looking for a FTW of (1) is equivalent to finding a solution of the following ODE systems

* Supported by the National Natural Science Foundation of China 19471024 and the Natural Science Foundation of Jiangsu Province. 


$$
\left\{\begin{array}{l}
d_{i} y_{i}^{\prime \prime}=c y_{i}^{\prime}+e_{i} \prod_{j=1}^{n} y_{j}^{m_{i j}}, z>0, c \in \mathbb{R}^{1}, \\
y_{i}(0)=y_{i}^{\prime}(0)=0, \\
y_{i}(z) \geq 0 \text { for } z>0, \text { and } y_{i}(z)>0 \text { for } z>0 \text { and close to } 0, i=1, \ldots, n,
\end{array}\right.
$$

where' $=d / d z$.

We can prove that the solution $\left(y_{1}(z), \ldots, y_{n}(z)\right)$ of (2) satisfies $y_{i}(z)>0$ for $z>0$, $i=1, \ldots, n$. Therefore, $y_{i}^{\prime}(z)>0$ for $z>0$ and $y_{i}(z) \in C^{2}\left(0, z^{*}\right) \cap C^{1}\left[0, z^{*}\right), i=1, \ldots, n$, where $z^{*}<+\infty$ or $z^{*}=+\infty$ is the maximum existence time of $\left(y_{1}(z), \ldots, y_{n}(z)\right)$. It follows that the line $x=-c t$ is a front separating the region $P_{+}\left(u_{1}, \ldots, u_{n}\right)=$ $\left\{(x, t) \mid u_{i}(x, t)>0, i=1, \ldots, n\right\}$ from the one where $u_{i}(x, t)=0, i=1, \ldots, n$.

By the standard theory of ordinary differential equations we can prove that

(a) $\lim _{z \rightarrow z^{*}} \sum_{i=1}^{n} y_{i}(z)=+\infty$ if $z^{*}<+\infty$;

(b) $\lim _{z \rightarrow z^{*}} y_{i}(z)=\lim _{z \rightarrow z^{*}} y_{i}^{\prime}(z)=+\infty$ if $z^{*}=+\infty, 1 \leq i \leq n$.

In fact, by the continuation of solutions theory the conclusion (a) is obvious. To prove conclusion (b), let $f_{i}(z)=\prod_{j=1}^{n} y_{j}^{m_{i j}}(z)$. Because $y_{i}(z)>0$ and $y_{i}^{\prime}(z)>0$, we have that $f_{i}^{\prime}(z) \geq 0$ and there exists $f_{0}>0$ such that $f_{i}(z) \geq f_{0}$ for all $z \geq 1$ and $1 \leq i \leq n$. By (2) we have

$$
\left\{\begin{array}{l}
y_{i}^{\prime \prime}=\frac{c}{d_{i}} y_{i}^{\prime}+\frac{e_{i}}{d_{i}} f_{i} \geq \frac{c}{d_{i}} y_{i}^{\prime}+\frac{e_{i}}{d_{i}} f_{0}, z \geq 1 \\
y_{i}(z)>0, y_{i}^{\prime}(z)>0 \text { for } z \geq 1
\end{array}\right.
$$

If $c=0$, then we have $y_{i}^{\prime}(z) \geq \frac{e_{i}}{d_{i}} f_{0}(z-1) \rightarrow+\infty$ as $z \rightarrow+\infty$ and hence $y_{i}(z) \rightarrow+\infty$ as $z \rightarrow+\infty, 1 \leq i \leq n$.

If $c \neq 0$, then we have

$$
y_{i}^{\prime}(z) \geq y_{i}^{\prime}(1) \exp \left\{\frac{c}{d_{i}}(z-1)\right\}+\frac{e_{i}}{c} f_{0}\left(\exp \left\{\frac{c}{d_{i}}(z-1)\right\}-1\right)
$$

Therefore $y_{i}^{\prime}(z) \geq \tau_{i} f_{0}$ for some $\tau_{i}>0$ and $z \geq 2$. Hence $y_{i}(z) \rightarrow+\infty$ as $z \rightarrow+\infty$, $1 \leq i \leq n$. Because $\sum_{j=1}^{n} m_{i j}>0$, we have that $f_{i}(z) \rightarrow+\infty$ as $z \rightarrow+\infty$. Therefore

$$
\begin{aligned}
y_{i}^{\prime}(z) & =y_{i}^{\prime}\left(z_{0}\right) \exp \left\{\frac{c}{d_{i}}\left(z-z_{0}\right)\right\}+\frac{e_{i}}{d_{i}} \exp \left\{\frac{c}{d_{i}} z\right\} \int_{z_{0}}^{z} f_{i}(z) \exp \left\{-\frac{c}{d_{i}} z\right\} d z \\
& \geq \frac{e_{i}}{d_{i}} f_{i}\left(z_{0}\right) \exp \left\{\frac{c}{d_{i}} z\right\} \int_{z_{0}}^{z} \exp \left\{-\frac{c}{d_{i}} z\right\} d z \\
& \geq \frac{e_{i}}{d_{i}} f_{i}\left(z_{0}\right) \exp \left\{\frac{c}{d_{i}} z\right\} \frac{d_{i}}{c}\left[\exp \left\{-\frac{c}{d_{i}} z_{0}\right\}-\exp \left\{-\frac{c}{d_{i}} z\right\}\right] \\
& =\frac{e_{i}}{c} f_{i}\left(z_{0}\right)\left[\exp \left\{\frac{c}{d_{i}}\left(z-z_{0}\right)\right\}-1\right]
\end{aligned}
$$


for any $z>z_{0}>1$. Because $f_{i}\left(z_{0}\right) \rightarrow+\infty$ as $z_{0} \rightarrow+\infty$, we know that $y_{i}^{\prime}(z) \rightarrow+\infty$ as $z \rightarrow+\infty$.

Finite travelling waves of semilinear parabolic systems (1) were first studied by $\mathbf{J}$. Esquinas and M. A. Herrero in [2] for the case $n=2$ and $m_{11}=m_{22}=0$, $d_{1}=d_{2}=e_{1}=e_{2}=1$ by using the theory of integral equations and the Schauder fixed point theorem.

For the following quasilinear parabolic systems

$$
\left\{\begin{array}{l}
\left(u^{0}\right)_{t}=u_{x x}-u^{\alpha} v^{\beta}, \\
\left(v^{m}\right)_{t}=v_{x x}-u^{\rho} v^{q}
\end{array}\right.
$$

where $\theta, m>0$ and $\alpha, \beta, p, q \geq 0, \alpha+\beta>0, p+q>0$. In paper [5], we discussed the necessary and sufficient conditions on existence and large time behaviours of FTW of (3) by using an upper and lower solutions method. For the special case $\theta=m=1$, asymptotic behaviours of FTW of (3) as $z \rightarrow 0^{+}$and $z \rightarrow+\infty$ were given in [6] by using the similar method to that of [5].

Denote $M=\left(m_{i j}\right)_{n \times n}, A=I-M, I$ is the unit matrix. In this paper, we will prove that (1) has FTW if and only if all principal minors of $A$ are positive, and give some asymptotic behaviours of FTW as $z \rightarrow 0^{+}$and $z \rightarrow+\infty$. Our main results read as follows.

Theorem 1. Given $c \in \mathbb{R}^{\prime},(2)$ has at most one solution.

Theorem 2. For any $c \in \mathbb{R}^{\prime}$, (2) has a solution if and only if all principal minors of $A$ are positive.

Theorem 3. Let $\left(y_{1}(z), \ldots, y_{n}(z)\right)$ be the solution of (2). For any $c \in \mathbb{R}^{1}, y_{i}(z) \approx b_{i} z^{2 k_{i}}$ as $z \rightarrow 0^{+}, i=1, \ldots, n$. Where $k=\left(k_{1}, \ldots, k_{n}\right)^{T}$, with $k_{i}>1$, is the unique solution of the linear algebraic system

$$
A k=(1, \ldots, 1)^{T}
$$

and $b=\left(b_{1}, \ldots, b_{n}\right)$ is the unique positive solution $\left(i . e . b_{i}>0\right)$ of the nonlinear algebraic system

$$
\prod_{j=1}^{n} b_{j}^{m_{i j}}=\frac{1}{e_{i}} 2 k_{i}\left(2 k_{i}-1\right) d_{i} b_{i}, \quad i=1, \ldots, n .
$$

Theorem 4. Let $\left(y_{1}(z), \ldots, y_{n}(z)\right)$ be the solution of (2). If $c<0$, then $y_{i}(z) \approx D_{i} z^{k_{i}}$ as $z \rightarrow+\infty, i=1, \ldots, n$. Here $D=\left(D_{1}, \ldots, D_{n}\right)$ is the unique positive solution of the nonlinear algebraic system 


$$
\prod_{j=1}^{n} D_{j}^{m_{i j}}=-\frac{1}{e_{i}} c k_{i} D_{i}, i=1, \ldots, n .
$$

Theorem 5. Let $\left(y_{1}(z), \ldots, y_{n}(z)\right)$ be the solution of (2). If $c>0$ and $\sum_{j=1}^{n}\left(m_{i j} / d_{j}\right)<$ $1 / d_{i}$ for all $1 \leq i \leq n$, then $y_{i}(z)=O\left(e^{c_{i} z}\right)$ as $z \rightarrow+\infty$, where $c_{i}=c / d_{i}, i=1, \ldots, n$.

Here $y(z) \approx v(z)$ means that $\lim (y(z) / v(z))=1 ; y(z)=O(v(z))$ means that there exists $0<C<+\infty$ such that $\lim (y(z) / v(z))=C$.

\section{The preliminaries}

This section contains two parts. In the first one, we give some results on algebraic systems. In the second one, we state the upper and lower solutions method.

Proposition 1 ([1]). The $n \times n$ matrix $A=\left(a_{i j}\right)$ with $a_{i j} \leq 0$ for $i \neq j$ is called $a$ nonsingular $M$-matrix if it has one of the following equivalent properties:

(1) $A$ is nonsingular and $A^{-1} \geq 0$ (componentwise).

(2) All principal minors of $A$ are positive.

(3) All leading principal minors of $A$ are positive.

(4) $\operatorname{Re} \lambda>0$ for each eigenvalue $\lambda$ of $A$.

By this proposition we have the following lemmas.

Lemma 1. Assume that all principal minors of $A$ are positive. Then the linear algebraic system (4) has a unique solution $k=\left(k_{1}, \ldots, k_{n}\right)^{T}$ and satisfies $k_{i}>1, i=1, \ldots, n$.

Proof. By Proposition $1, A$ is nonsingular and $A^{-1} \geq 0$ (componentwise). Therefore, equation (4) has a unique solution $k=A^{-1}(1, \ldots, 1)^{T} \geq 0$, and

$$
\sum_{j=1}^{n} a_{i j} k_{j}=\left(1-m_{i i}\right) k_{i}-\sum_{j \neq i} m_{i j} k_{j}=1, i=1, \ldots, n .
$$

This yields $k_{i}>1$ for all $1 \leq i \leq n$ since $k_{i} \geq 0,0 \leq m_{i i}<1, m_{i j} \geq 0$ and $\sum_{j=1}^{n} m_{i j}>0$.

Lemma 2. Assume that all principal minors of $A$ are positive. Let $k=\left(k_{1}, \ldots, k_{n}\right)^{T}$ be the unique solution of (4) $\left(k_{i}>1\right)$. Then the nonlinear algebraic systems (5) and (6) have unique positive solution $b=\left(b_{1}, \ldots, b_{n}\right)^{T}$ and $D=\left(D_{1}, \ldots, D_{n}\right)^{T}$ respectively.

Proof. Denote $\alpha_{i}=2 k_{i}\left(2 k_{i}-1\right) d_{i} / e_{i}$, and let $\beta_{1}=\log b_{i}, \gamma_{i}=\log \alpha_{i}$. Then (5) is 
equivalent to

$$
A \beta=-\gamma
$$

where $\beta=\left(\beta_{1}, \ldots, \beta_{n}\right)^{T}$ and $\gamma=\left(\gamma_{1}, \ldots, \gamma_{n}\right)^{T}$. Equation (7) has a unique solution $\beta=-A^{-1} \gamma$. Hence (5) has a unique positive solution $b=\left(b_{1}, \ldots, b_{n}\right)^{T}$ with $b_{i}=e^{\beta_{i}}$, $i=1, \ldots, n$. Similarly, (6) has a unique positive solution $D=\left(D_{1}, \ldots, D_{n}\right)^{T}$.

Lemma 3. Assume that all principal minors of $A$ are positive and positive constants $b_{i}, b_{i}^{\prime}, x_{i}, x_{i}^{\prime}(i=1, \ldots, n)$ satisfy

$$
b_{i}=\alpha_{i} \prod_{j=1}^{n} b_{j}^{m_{i j}}, b_{i}^{\prime}=\alpha_{i}^{\prime} \prod_{j=1}^{n}\left(b_{j}^{\prime}\right)^{m_{i j}}, i=1, \ldots, n .
$$

If $\alpha_{i} \leq(<) \alpha_{i}^{\prime}$, then $b_{i} \leq(<) b_{i}^{\prime}$.

Proof. Let $\beta_{i}=\log b_{i}, \beta_{i}^{\prime}=\log b_{i}^{\prime}, \gamma_{i}=\log \alpha_{i}$ and $\gamma_{i}^{\prime}=\log \alpha_{i}^{\prime}, i=1, \ldots, n$. Then (8) is equivalent to

$$
A \beta=\gamma, A \beta^{\prime}=\gamma^{\prime},
$$

where $\beta=\left(\beta_{1}, \ldots, \beta_{n}\right)^{T}, \quad \beta^{\prime}=\left(\beta_{1}^{\prime}, \ldots, \beta_{n}^{\prime}\right)^{T}, \quad \gamma=\left(\gamma_{1}, \ldots, \gamma_{n}\right)^{T}, \quad \gamma^{\prime}=\left(\gamma_{1}^{\prime}, \ldots, \gamma_{n}^{\prime}\right)^{T} . \quad$ If $\alpha_{i} \leq(<) \alpha_{i}^{\prime}$, then $\gamma_{i} \leq(<) \gamma_{i}^{\prime}, i=1, \ldots, n$, and in turn

$$
A\left(\beta-\beta^{\prime}\right)=\gamma-\gamma^{\prime} \leq(<) 0 \text { (componentwise). }
$$

Since $A$ is an $M$-matrix, we have $\beta-\beta^{\prime} \leq(<) 0$ and in turn $b_{i} \leq(<) b_{i}^{\prime}, i=1, \ldots, n$.

Lemma 4. Assume that all principal minors of $A$ are positive and $c<0$. Let $D=\left(D_{1}, \ldots, D_{n}\right)^{T}$ be the unique positive solution of (6). Then there exists a sequence $\left\{D^{(p)}=\left(D_{1}^{(p)}, \ldots, D_{n}^{(p)}\right)^{T}\right\}$, with $D_{i}^{(p)}>0$, such that

$$
\begin{aligned}
& \prod_{j=1}^{n}\left(D_{j}^{(p)}\right)^{m_{i j}}>-\frac{c}{e_{i}} k_{i} D_{i}^{(p)}, D_{i}^{(p)}<D_{i} \text { and } \\
& D_{i}^{(p)} \rightarrow D_{i} \text { as } p \rightarrow+\infty, i=1, \ldots, n .
\end{aligned}
$$

Proof. Denote $\alpha_{i}=\left(-c k_{i} / e_{i}\right)^{-1}$. Then $D$ and $\alpha=\left(\alpha_{1}, \ldots, \alpha_{n}\right)^{T}$ satisfy

$$
\alpha_{i} \prod_{j=1}^{n} D_{j}^{m_{i j}}=D_{i}, i=1, \ldots, n
$$

Choose $\alpha_{i}^{(p)}$ such that $0<\alpha_{i}^{(p)}<\alpha_{i}$ and $\alpha_{i}^{(p)} \rightarrow \alpha_{i}$ as $p \rightarrow+\infty$. Let $D^{(p)}=$ 
$\left(D_{1}^{(p)}, \ldots, D_{n}^{(p)}\right)^{T}$ be the unique positive solution of

$$
\alpha_{i}^{(p)} \prod_{j=1}^{n}\left(D_{j}^{(p)}\right)^{m_{i j}}=D_{i}^{(p)}, i=1, \ldots, n .
$$

Then we have

$$
\prod_{j=1}^{n}\left(D_{j}^{(p)}\right)^{m_{i j}}=\frac{1}{\alpha_{i}^{(p)}} D_{i}^{(p)}>\frac{1}{\alpha_{i}} D_{i}^{(p)}=-\frac{c}{e_{i}} k_{i} D_{i}^{(p)},
$$

and $D_{i}^{(p)}<D_{i}$ by Lemma 3. Since $\alpha_{i}^{(p)} \rightarrow \alpha_{i}$, by continuity, we have $D_{i}^{(p)} \rightarrow D_{i}$ as $p \rightarrow+\infty, i=1, \ldots, n$.

Upper and lower solutions method. Assume that $\underline{y}_{i}, \bar{y}_{i} \in C^{2}[0, \varepsilon]$ are positive functions for some $\varepsilon>0$, and satisfy

$$
\begin{aligned}
& d_{i} \underline{y}_{i}^{\prime \prime}-c \underline{y}_{i}^{\prime}-e_{i} \prod_{j=1}^{n} \underline{y}_{j}^{m_{i j}} \leq 0 \leq d_{i} \bar{y}_{i}^{\prime \prime}-c \bar{y}_{i}^{\prime}-e_{i} \prod_{j=1}^{n} \bar{y}_{j}^{m_{j j}} \text { in }[0, \varepsilon], \\
& \underline{y}_{i}(0)=0 \leq \bar{y}_{i}(0), \underline{y}_{i}^{\prime}(0)=0 \leq \bar{y}_{i}^{\prime}(0), \underline{y}_{i}(z) \leq \bar{y}_{i}(z) \text { in }[0, \varepsilon], i=1, \ldots, n .
\end{aligned}
$$

Then system (2) has a unique positive solution $y=\left(y_{1}, \ldots, y_{n}\right)$ and satisfies $\underline{y}_{i} \leq y_{i} \leq \bar{y}_{i}$ in $[0, \varepsilon], i=1, \ldots, n$. Here $\underline{y}=\left(\underline{y}_{1}, \ldots, \underline{y}_{n}\right)$ and $\bar{y}=\left(\bar{y}_{1}, \ldots, \bar{y}_{n}\right)$ are called the ordered lower and upper solutions of (2).

Existence of $y$ can be proved by the standard iterative techniques because system (2) is quasimonotone increasing, cf. $[3,4,6]$. The uniqueness can be proved as in [5].

\section{Proofs of theorems}

It is obvious that (2) is equivalent to the following integral differential system

$$
\left\{\begin{array}{l}
y_{i}^{\prime}(z)=\frac{c}{d_{i}} y_{i}(z)+\frac{e_{i}}{d_{i}} \int_{0}^{z} \prod_{j=1}^{n} y_{j}^{m_{i j}}(s) d s, z>0, \\
y_{i}(0)=0, y_{i}(z)>0 \text { for } z>0, i=1, \ldots, n
\end{array}\right.
$$

and it is also equivalent to the following integral system

$$
\left\{\begin{array}{l}
y_{i}(z)=\frac{e_{i}}{c} \int_{0}^{z}\left[\exp \left\{\frac{c}{d_{i}}(z-s)\right\}-1\right] \prod_{j=1}^{n} y_{j}^{m_{i j}}(s) d s, \text { if } c \neq 0 \\
y_{i}(z)=\frac{e_{i}}{d_{i}} \int_{0}^{z}(z-s) \prod_{j=1}^{n} y_{j}^{m_{i j}}(s) d s, \text { if } c=0 \\
y_{i}(z)>0 \text { for } z>0, i=1, \ldots, n
\end{array}\right.
$$

The proof of Theorem 1 is the same as that of the uniqueness in paper [5]. 
Proof of Theorem 2. We first prove the necessity. Assume that $\left(y_{1}(z), \ldots, y_{n}(z)\right)$ is a solution of (2), then it satisfies (10), (11). Since $y_{i}(z)>0$ and $y_{i}^{\prime}(z)>0, i=1, \ldots, n$, from (11) it follows that, for some positive constant $C$ and small positive constant $z_{0}$,

$$
y_{i}(z) \leq C z^{2} \prod_{j=1}^{n} y_{j}^{m_{i j}}(z), \text { for } 0<z \leq z_{0}, i=1, \ldots, n .
$$

We will focus our attention on the inequalities (12). Taking the number of inequalities of (12) as an induction variable, we use the mathematical-induction method to complete the proof. When $n=1$, it is obvious that $a_{11}=1-m_{11}>0$ because $y_{1}(z) \rightarrow 0$ as $z \rightarrow 0^{+}$. Assume that the conclusion holds for $n-1$. Then for $n$, using $y_{i}(0)=0$ and $y_{i}^{\prime}(z)>0$ we have that $y_{i}(z) \leq \sigma$ for all $0 \leq z \leq z_{0}$ and some $\sigma>0, i=1, \ldots, n$. From (12) it follows that, for any $1 \leq l \leq n$,

$$
y_{i}(z) \leq C \sigma^{m_{i} l} z^{2} \prod_{j \neq l} y_{j}^{m_{i j}}(z), \text { for } 0 \leq z \leq z_{0} \text { and } i=1, \ldots, n, i \neq l .
$$

For any fixed $l: 1 \leq l \leq n$, because the number of inequalities in (13) is $n-1$, by the inductive assumption we have that all $p$-th order principal minors of $A=I-M$ are positive for $p=1, \ldots, n-1$. In particular $a_{n n}>0$. From (12) we have

$$
y_{n}(z) \leq\left(C z^{2}\right)^{1 / a_{n n}} \prod_{j=1}^{n-1} y_{j}^{m_{n j} / a_{n n}}(z), 0 \leq z \leq z_{0} .
$$

Hence

$$
y_{i}(z) \leq\left(C z^{2}\right)^{1+m_{i n} / a_{n n}} \prod_{j=1}^{n-1} y^{m_{i j}+m_{i n} m_{n j} / a_{n n}}(z), 0 \leq z \leq z_{0}, i=1, \ldots, n-1
$$

Denote $m_{i j}^{(n)}=m_{i j}+m_{i n} m_{n j} / a_{n n}, a_{i j}^{(n)}=a_{i j}-a_{i n} a_{n j} / a_{n n}, i, j=1, \ldots, n-1, \widetilde{M}=\left(m_{i j}^{(n)}\right)_{(n-1) \times(n-1)}$, $\widetilde{A}=\left(a_{i j}^{(n)}\right)_{(n-1) \times(n-1)}$, then we have $m_{i j}^{(n)} \geq 0, \widetilde{A}=I-\widetilde{M}$ and $\operatorname{det} A=a_{n n} \operatorname{det} \widetilde{A}$. Because the number of inequalities in (14) is $n-1$, by the inductive assumption it follows that $\operatorname{det} \tilde{A}>0$, and hence $\operatorname{det} A>0$. This shows that all principal minors of $A$ are positive. The proof of the necessity is completed.

In the following we prove the sufficiency of Theorem 2 . We assume that all principal minors of $A$ are positive. Using Lemmas 1 and 2 we have that the algebraic system (4) has a unique solution $k=\left(k_{1}, \ldots, k_{n}\right)^{T}$ with $k_{i}>1, i=1, \ldots, n$, and the algebraic system (5) has a unique positive solution $b=\left(b_{1}, \ldots, b_{n}\right)^{T}\left(b_{i}>0, i=1 \ldots, n\right)$.

(1) The Case $c=0$. Let $y_{i}(z)=b_{i} z^{2 k_{i}}, i=1 \ldots, n$. Using (4) and (5) we can verify that $\left(y_{1}(z), \ldots, y_{n}(z)\right)$ is a solution of (2).

(2) The Case $c \neq 0$. Choose $0<\sigma<e_{i} / 2$ and let $\underline{y}_{i}=\underline{b}_{i} z^{2 k_{i}}, \bar{y}_{i}=\bar{b}_{i} z^{2 k_{i}}, i=1 \ldots, n$, where $\left(\underline{b}_{1}, \ldots, \underline{b}_{n}\right)^{T}$ and $\left(\bar{b}_{1}, \ldots, \bar{b}_{n}\right)^{T}$ are solutions of 


$$
\prod_{j=1}^{n} \underline{b}_{j}^{m_{i j}}=\frac{1}{e_{i}-\sigma} 2 k_{i}\left(2 k_{i}-1\right) d_{i} \underline{b}_{i}, i=1 \ldots, n
$$

and

$$
\prod_{j=1}^{n} \bar{b}_{j}^{m_{i j}}=\frac{1}{e_{i}+\sigma} 2 k_{i}\left(2 k_{i}-1\right) d_{i} \bar{b}_{i}, i=1 \ldots, n
$$

respectively. Then we have (similar to Case 1)

$$
\begin{aligned}
& d_{i} \underline{y}_{i}^{\prime \prime}=\left(e_{i}-\sigma\right) \prod_{j=1}^{n} \underline{y}_{j}^{m_{i j}}, z>0, \\
& d_{i} \bar{y}_{i}^{\prime \prime}=\left(e_{i}+\sigma\right) \prod_{j=1}^{n} \bar{y}_{j}^{m_{i j}}, z>0, \\
& \underline{y}_{i}(0)=\bar{y}_{i}(0)=\underline{y}_{i}^{\prime}(0)=\bar{y}_{i}^{\prime}(0)=0, \\
& i=1, \ldots, n .
\end{aligned}
$$

Since the power of $z$ in $\underline{y}_{i}^{\prime}\left(\bar{y}_{i}^{\prime}\right)$ is $2 k_{i}-1$ and the powers of $z$ in $\underline{y}_{i}^{\prime \prime}$ and $\prod_{j=1}^{n} \underline{y}_{j}^{m_{i j}}\left(\bar{y}_{i}^{\prime \prime}\right.$ and $\prod_{j=1}^{n} \bar{y}_{j}^{m_{i j}}$ ) are $2 k_{i}-2$, it follows that there exists $\varepsilon>0$, depending only on $c$ and $\sigma$, such that

$$
\begin{aligned}
& d_{i} \underline{y}_{i}^{\prime \prime} \leq c \underline{y}_{i}^{\prime}+e_{i} \prod_{j=1}^{n} \underline{y}_{j}^{m_{i j}} \text { in }[0, \varepsilon], i=1, \ldots, n, \\
& d_{i} \bar{y}_{i}^{\prime \prime} \geq c \bar{y}_{i}^{\prime}+e_{i} \prod_{j=1}^{n} \bar{y}_{j}^{m_{i j}} \text { in }[0, \varepsilon], i=1, \ldots, n .
\end{aligned}
$$

This shows that $\underline{y}=\left(\underline{y}_{1}, \ldots, \underline{y}_{n}\right)$ and $\bar{y}=\left(\bar{y}_{1}, \ldots, \bar{y}_{n}\right)$ are the ordered lower and upper solutions of $(2)$ in $[0, \bar{\varepsilon}]$. Therefore, $(2)$ has a unique positive solution $y=\left(y_{1}, \ldots, y_{n}\right)$ in $[0, \varepsilon]$ and satisfies

$$
\underline{b}_{i} z^{2 k_{i}}=\underline{y}_{i} \leq y_{i} \leq \bar{y}_{i}=\bar{b}_{i} z^{2 k_{i}} \text { in }[0, \varepsilon], i=1, \ldots, n
$$

Let $z^{*}$ be the maximal existence time of $\left(y_{1}(z), \ldots, y_{n}(z)\right)$, and

$$
F_{i}(z)=\frac{e_{i}}{c} \int_{0}^{z}\left(\exp \left\{\frac{c}{d_{i}}(z-s)\right\}-1\right) d s, i=1, \ldots, n,
$$

then $F_{i}(z)$ is a continuous positive function in $(0,+\infty)$. Using $y_{i}(z)>0, y_{i}^{\prime}(z)>0$ and 
(11), it follows that $y_{i}(z) \leq F_{i}(z) \prod_{j=1}^{n} y_{j}^{m_{i j}}(z), 0<z<z^{*}, i=1, \ldots, n$. Lemma 3 shows that $y_{i}(z)$ is bounded in $\left(0, z^{*}\right)$ if $z^{*}<+\infty, i=1, \ldots, n$. Consequently, $z^{*}=+\infty$ and $\left(y_{1}(z), \ldots, y_{n}(z)\right)$ is a global solution of $(2)$.

Theorem 2 is proved.

Proof of Theorem 3. Assume that $\left(y_{1}(z), \ldots, y_{n}(z)\right)$ is a solution of (2). By Theorem 2 we have that all principal minors of $A$ are positive.

(1) If $c=0$, by the uniqueness of solution of (2), it follows that $y_{i}(z)=b_{i} z^{2 k_{i}}, z>0$, $i=1, \ldots, n$. Hence the conclusion holds.

(2) If $c \neq 0$, by the uniqueness of solution of (2) we know that $y_{i}(z)$ satisfies (15). Let $\sigma \rightarrow 0$ and $z \rightarrow 0^{+}$, it follows that $y_{i}(z) \approx b_{i} z^{2 k_{i}}$ as $z \rightarrow 0^{+}$since $\underline{b}_{i}, \bar{b}_{i} \rightarrow b_{i}$ as $\sigma \rightarrow 0$, $i=1, \ldots, n$.

The proof of Theorem 3 is completed.

Proof of Theorem 4. Let $\left(y_{1}(z), \ldots, y_{n}(z)\right)$ be a solution of (2) and $c<0$. By Theorem 2 we have that all principal minors of $A$ are positive. By Lemmas 2 and $4,(6)$ has a unique positive solution $D=\left(D_{1}, \ldots, D_{n}\right)$, and there exist $D^{(p)}=\left(D_{1}^{(p)}, \ldots, D_{n}^{(p)}\right)$, $p=1,2, \ldots$, such that (9) holds.

Let $y_{i}^{\prime}=v_{i}(z)$, then (2) is equivalent to

$$
\left\{\begin{array}{l}
y_{i}^{\prime}(z)=v_{i}(z), z>0 \\
d_{i} v_{i}^{\prime}(z)=c v_{i}(z)+e_{i} \prod_{j=1}^{n} y_{j}^{m_{i j}}(z), z>0 \\
y_{i}(0)=v_{i}(0)=0, y_{i}(z), v_{i}(z)>0 \text { for } z>0, i=1, \ldots, n .
\end{array}\right.
$$

Using (4) and (6) we have that, for any $\varepsilon>0$,

$$
d_{i} D_{i} k_{i}\left(k_{i}-1\right)(z+\varepsilon)^{k_{i}-2} \geq c D_{i} k_{i}(z+\varepsilon)^{k_{i}-1}+e_{i} \prod_{j=1}^{n} D_{j}^{m_{i j}}(z+\varepsilon)^{m_{i j} k_{j}}=0, z>0, i=1, \ldots, n
$$

Let $\bar{y}_{i}(z)=D_{i}(z+\varepsilon)^{k_{i}}$ and $\bar{v}_{i}(z)=D_{i} k_{i}(z+\varepsilon)^{k_{i}-1}, i=1, \ldots, n$. By (17) we know that $\left(\bar{y}_{1}(z), \ldots, \bar{y}_{n}(z), \bar{v}_{1}(z), \ldots, \bar{v}_{n}(z)\right)$ is an upper solution of (16). By the comparison principle (see [3]) we get $y_{i}(z) \leq D_{i}(z+\varepsilon)^{k_{i}}, \quad y_{i}^{\prime}(z)=v_{i}(z) \leq D_{i} k_{i}(z+\varepsilon)^{k_{i}-1}, \quad z \geq 0$, $i=1, \ldots, n$. Thus we have

$$
\lim _{z \rightarrow+\infty} \sup \left(y_{i}(z) / z^{k_{i}}\right) \leq D_{i}, i=1, \ldots, n
$$

Using (4) and (9), it follows that there exists $z_{1}^{(p)} \gg 1$ such that

$$
d_{i} D_{i}^{(p)} k_{i}\left(k_{i}-1\right) z^{k_{i}-2} \leq c D_{i}^{(p)} k_{i} z^{k_{i}-1}+e_{i} \prod_{j=1}^{n}\left(D_{j}^{(p)}\right)^{m_{i j}} z^{m_{i j} k_{j}}, z \geq z_{1}^{(p)}, i=1, \ldots, n .
$$


Since $y_{i}(z) \rightarrow+\infty, y_{i}^{\prime}(z) \rightarrow+\infty$ as $z \rightarrow+\infty$, there exists $z_{2}^{(p)}>z_{1}^{(p)}$, such that

$$
y_{i}\left(z_{2}^{(p)}\right) \geq D_{i}^{(p)}\left(z_{1}^{(p)}\right)^{k_{i}}, y_{i}^{\prime}\left(z_{2}^{(p)}\right) \geq D_{i}^{(p)} k_{i}\left(z_{1}^{(p)}\right)^{k_{i}-1}, i=1, \ldots, n
$$

Let $\underline{y}_{i}(z)=D_{i}^{(p)}\left(z-z_{2}^{(p)}+z_{1}^{(p)}\right)^{k_{i}}$ and $\underline{v}_{i}(z)=D_{i}^{(p)} k_{i}\left(z-z_{2}^{(p)}+z_{1}^{(p)}\right)^{k_{i}-1}, \quad i=1, \ldots, n$. Using (16), (19) and (20), it follows by the comparison principle that (see [3])

$$
y_{i}(z) \geq \underline{y}_{i}(z) \geq D_{i}^{(p)}\left(z-z_{2}^{(p)}+z_{1}^{(p)}\right)^{k_{i}}, \text { for } z \geq z_{2}^{(p)}, i=1, \ldots, n ; p=1,2, \ldots
$$

Since $\lim _{z \rightarrow+\infty}\left(z-z_{2}^{(p)}+z_{1}^{(p)}\right)^{k_{i}} / z^{k_{i}}=1$, there exists $z_{3}^{(p)}>z_{2}^{(p)}$ such that

$$
D_{i}^{(p)}\left(z-z_{2}^{(p)}+z_{1}^{(p)}\right)^{k_{i}} \geq\left(D_{i}^{(p)}-\frac{1}{p}\right) z^{k_{i}} \text { for } z \geq z_{3}^{(p)}, i=1, \ldots, n ; p=1,2, \ldots
$$

Therefore

$$
y_{i}(z) \geq\left(D_{i}^{(p)}-\frac{1}{p}\right) z^{k_{i}} \text { for } z \geq z_{3}^{(p)}, i=1, \ldots, n ; p=1,2, \ldots
$$

Let $p \rightarrow+\infty$, using $D_{i}^{(p)} \rightarrow D_{i}$, we have $\lim _{z \rightarrow+\infty} \inf \left(y_{i}(z) / z^{k_{i}}\right) \geq D_{i}, i=1, \ldots, n$. This fact, combined with (18), yields that Theorem 4 holds.

Proof of Theorem 5. Let $\left(y_{1}(z), \ldots, y_{n}(z)\right)$ be a solution of (2) and $c>0$. By Theorem 2 we have that all principal minors of $A$ are positive. Let $c_{i}=c / d_{i}$, $w_{i}(z)=y_{i}(z) e^{-c_{i} z}$, then we have

$$
\begin{gathered}
w_{i}^{\prime}(z)=\frac{e_{i}}{d_{i}} e^{-c_{i} z} \int_{0}^{z} \prod_{j=1}^{n} y_{j}^{m_{i j}}(s) d s>0, z>0, i=1, \ldots, n . \\
w_{i}(z)=\frac{e_{i}}{c} \int_{0}^{z}\left(e^{-c_{i} s}-e^{-c_{i} z}\right)\left(\prod_{j=1}^{n} w_{j}^{m_{i j}}(s)\right) \exp \left\{\left(\sum_{j=1}^{n} m_{i j} c_{j}\right) s\right\} d s, i=1, \ldots, n .
\end{gathered}
$$

Denote $\tau_{i}=c_{i}-\sum_{j=1}^{m} m_{i j} c_{j}$, by the assumption $\sum_{j=1}^{n}\left(m_{i j} / d_{j}\right)<1 / d_{i}$ we know that $\tau_{i}>0,1 \leq i \leq n$. Using (21) and (22) it follows that 


$$
\begin{aligned}
w_{i}(z) & \leq \frac{e_{i}}{c}\left(\prod_{j=1}^{n} w_{j}^{m_{i j}}(z)\right) \int_{0}^{z} e^{-c_{i j}} \exp \left\{\left(\sum_{j=1}^{n} m_{i j} c_{j}\right) s\right\} d s \\
& =\frac{e_{i}}{c}\left(\prod_{j=1}^{n} w_{j}^{m_{i j}}(z)\right) \int_{0}^{z} e^{-\tau_{i j} s} d s \\
& \leq \frac{e_{i}}{c \tau_{i}} \prod_{j=1}^{n} w_{j}^{m_{i j}}(z) \\
& \leq K \prod_{j=1}^{n} w_{j}^{m_{i j}}(z), z \geq 0, i=1, \ldots, n
\end{aligned}
$$

for some positive constant $K$. By Lemma 3 it follows that $w_{i}(z)$ is bounded in $[0,+\infty), \quad i=1, \ldots, n$. This fact, combined with $w_{i}^{\prime}(z) \geq 0$, yields that the limit $\lim _{z \rightarrow+\infty} w_{i}(z)=w_{i}$ exists and $0<w_{i}<+\infty$. Consequently, $y_{i}(z) \approx O\left(e^{c_{i} z}\right)$ as $z \rightarrow+\infty$, $i=1, \ldots, n$. The proof is completed.

Acknowledgement. I am very grateful to the referee for his (or her) many helpful comments which improved my presentation. Thank you.

\section{REFERENCES}

1. A. Berman and R. J. Plemmons, Nonnegative Matrices in the Mathematical Sciences (Academic Press, New York, 1979).

2. J. Esquinas and M. A. Herrero, Travelling wave solutions to semilinear diffusion system, SIAM J. Math. Anal. 21 (1990), 123-136.

3. G. S. LAdDe, V. Lakshmikantham and V. S. VatSala, Monotone Iterative Techniques for Nonlinear Differential Equations (Pitman, New York, 1985).

4. W. WALTER, Differential and Integral Inequalities (Springer-Verlag, 1970).

5. M. X. WANG, Existence of finite travelling waves for a nonlinear parabolic system, Nonlinear Anal., TMA 22 (1994), 1487-1500.

6. M. X. WANG, Nonlinear Equations of Parabolic Type (Science Press, Beijing, 1993), in Chinese.

Department of Applied Mathematics

SOUTHEAST UNIVERSITY

NANJING 210018

P.R. ChINA

E-mail address: mxwang@seu.edu.cn 